\title{
Analysis of Thermoluminescence Glow Peaks in $\beta$-Irradiated TlGaSeS Crystals
}

\author{
M. $\operatorname{ISIK}^{a, *}$, T. YILDIRIM ${ }^{b}$ AND N.M. GASANLY ${ }^{c, d}$ \\ ${ }^{a}$ Department of Electrical and Electronics Engineering, Atilim University, 06836 Ankara, Turkey \\ ${ }^{b}$ Department of Physics, Nevsehir University, 50300 Nevsehir, Turkey \\ ${ }^{c}$ Department of Physics, Middle East Technical University, 06800 Ankara, Turkey \\ ${ }^{d}$ Virtual International Scientific Research Centre, Baku State University, 1148 Baku, Azerbaijan
}

(Received April 1, 2015; in final form April 13, 2016)

Thermoluminescence properties of TlGaSeS layered single crystals were investigated in the temperature range of $280-720 \mathrm{~K}$. Thermoluminescence glow curve exhibited three peaks with maximum temperatures of $\approx 370,437$, and $490 \mathrm{~K}$. Curve fitting, initial rise and peak shape methods were used to determine the activation energies of the trapping centers. All applied methods resulted with energies around 0.82, 0.91, and $0.99 \mathrm{eV}$. Dose dependence of the thermoluminescence intensity was also examined for the doses in the range of 0.7-457.6 Gy. Peak maximum intensity of the observed peak around $370 \mathrm{~K}$ showed an increase up to a certain dose and then a decrease at higher doses. This non-monotonic dose dependence was discussed under the light of a reported model in which different kinds of competition between radiative and nonradiative recombination centers during excitation or heating stages of the thermoluminescence process are explained.

DOI: 10.12693/APhysPolA.129.1165

PACS/topics: 78.60.Kn, 71.55.Ak, 61.82.Fk

\section{Introduction}

The quaternary TlGaSeS crystal, member of IIIVI semiconductor group are formed from $\mathrm{TlGaS}_{2}$ and $\mathrm{TlGaSe}_{2}$ crystals by replacing half of the sulfur (selenium) atoms with selenium (sulfur) atoms [1,2]. They are promising for various technological applications, especially for optoelectronic device applications due to its high photosensitivity and components in the visible range of spectra and high birefringence in conjunction with a wide transparency range of $0.5-14.0 \mu \mathrm{m}[2-4]$. In the crystal structure of $\mathrm{TlGaSeS}, \mathrm{Tl}$ and $\mathrm{Se}(\mathrm{S})$ atoms are bonded with an interlayer type whereas bonding between $\mathrm{Ga}$ and $\mathrm{Se}(\mathrm{S})$ atoms is intralayer type. Optical indirect and direct band gap energies of TlGaSeS crystal were found experimentally as $2.27 \mathrm{eV}$ and $2.58 \mathrm{eV}$, respectively [5].

The influence of defects on the performance of optoelectronic devices is a well-known subject. In optoelectronic devices such as LEDs or lasers, defects may introduce nonradiative recombination centers which could lower the internal quantum efficiency or even render light generation impossible, depending on the defect density. In the case of electronic devices, the defects introduce scattering centers lowering carrier mobility, hence hindering high-frequency operation. Therefore, estimation of defect parameters in the crystals is important to fabricate better devices. Previously, TlGaSeS were characterized to get information about the donor, acceptor and/or

*corresponding author; e-mail: mehmet.isik@atilim.edu.tr trap levels in the crystal. Photoluminescence (PL) experiments performed in the wavelength region of 695$1010 \mathrm{~nm}$ and temperature range of $20-56 \mathrm{~K}$ revealed the presence of two PL bands [6]. Analysis showed that these two PL bands are associated with radiative transitions from deep donor levels located at 0.72 and $1.07 \mathrm{eV}$ below the bottom of the conduction band to shallow acceptor levels located at 8 and $11 \mathrm{meV}$ above the top of valence band. Thermally stimulated current (TSC) and thermoluminescence (TL) measurements below room temperature were performed on TlGaSeS to study the trapping centers in the crystal. TSC analysis showed that there exist three shallow levels at 13,20 , and $50 \mathrm{meV}$ [7]. In the TL experiments, two peaks associated with trapping centers at 16 and $97 \mathrm{meV}$ were observed [8].

To the best of our knowledge, high temperature thermoluminescence in TlGaSeS single crystals has not been reported in the literature yet. In this paper, we report the high temperature thermoluminescence properties by beta irradiation of TlGaSeS single crystals using a ${ }^{90} \mathrm{Sr}$ beta source in the 280-720 K temperature range. Thermal activation energies, capture cross-sections and attempt-toescape frequencies of the revealed trapping centers were obtained from basic techniques used for analysis of TL data.

\section{Experimental details}

TlGaSeS polycrystals were synthesized from highpurity elements (at least $99.999 \%$ ) prepared in stoichiometric proportions. Single crystals of TlGaSeS were grown by the Bridgman method in evacuated $\left(10^{-5}\right.$ Torr $)$ silica tubes with a tip at the bottom. The ampoule was moved in a vertical furnace through a thermal gradient of $30^{\circ} \mathrm{C} \mathrm{cm}^{-1}$, between the temperatures 850 and 
$480^{\circ} \mathrm{C}$ at a rate of $1.0 \mathrm{~mm} \mathrm{~h}^{-1}$. The resulting ingot appears red in color and the freshly cleaved surfaces were mirror-like. Chemical composition of the growth crystals was obtained from energy dispersive spectroscopy (EDS) measurements performed by JSM-6400 scanning electron microscope. Analysis of the EDS measurements revealed the atomic composition ratio of the constituent elements (Tl : Ga : Se : S) to be 25.7: 25.9: $24.3: 24.1$, respectively.

The TL glow curves were recorded with Ris $\varnothing \mathrm{TL} / \mathrm{OSL}$ DA-20 reader using Schott BG/39, $4 \mathrm{~mm}$ of thickness, and Corning $7 / 59,4 \mathrm{~mm}$ of thickness, optical filters. TL glow curves were obtained with heating at a constant heating rate of $5 \mathrm{~K} / \mathrm{s}$ up to $720 \mathrm{~K}$. The dose response curves of the sample exposed to ${ }^{90} \mathrm{Sr}$ beta radiation $(40 \mathrm{mCi})$ were obtained in the dose range from 0.7 to 457.6 Gy.

\section{Results and discussion}

Figure 1 shows the observed TL glow curves of TlGaSeS irradiated at different doses. In the figure, the temperature range in which TL peaks were observed is presented, although measurements were carried out in the 280-720 $\mathrm{K}$ temperature range. Three peaks with maximum temperatures $\left(T_{m}\right)$ of $\approx 370,437$, and $490 \mathrm{~K}$ are exhibited in the curves.

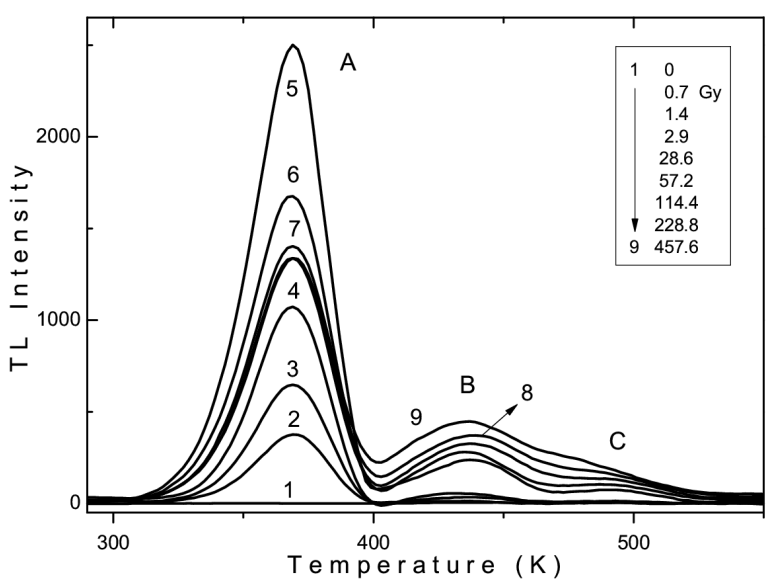

Fig. 1. TL curves of TlGaSeS crystals irradiated at different doses at a constant heating rate $\beta=5.0 \mathrm{~K} / \mathrm{s}$.

Dose dependence of TL intensities show differences for peak observed at $370 \mathrm{~K}$ (peak A) and other peaks (peak $\mathrm{B}$ and $\mathrm{C}$ ). TL intensities of peaks $\mathrm{B}$ and $\mathrm{C}$ increase with dose whereas peak A shows non-monotonic dose dependence in which TL intensity increases with dose up to a certain value and then decreases at higher doses by a factor of $\approx 0.53$ from the maximum (see Fig. 2).

Although such effect is described as "radiation effect" by many researchers in the literature, Lawless et al. reported a model for non-monotonic dose dependence of thermoluminescence [9]. Authors stated the main concept of this dependence as the different kinds of competitions taking place in the excitation and heating stages

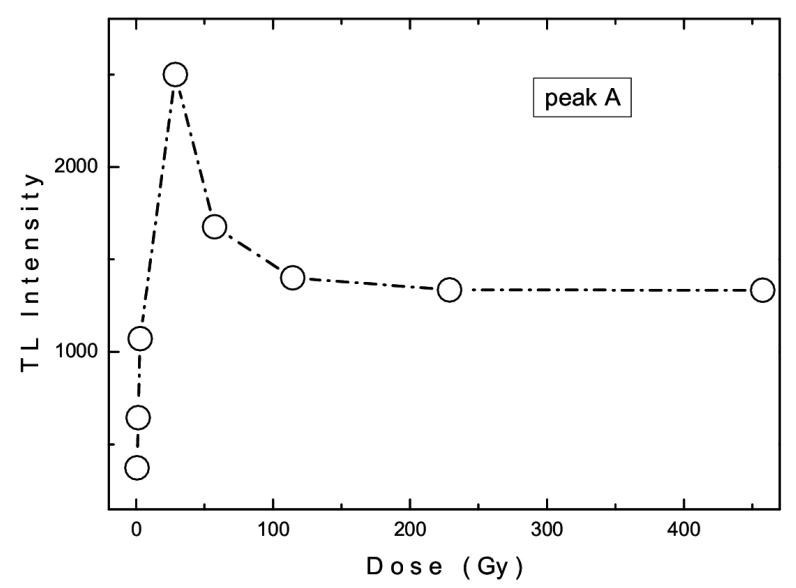

Fig. 2. Dose dependence of maximum TL intensities of peak $\mathrm{A}$.

of the TL process. The energy level diagram showing main transitions during excitation and heating is shown in Fig. 3. One trapping center and two kinds of recombination centers are presented to describe the model. In the figure, $P_{1}, P_{2}$ and $N$ are concentrations of non-radiative hole centers, radiative hole centers, and electron trapping states, respectively, with instantaneous occupancies of $p_{1}, p_{2}$ and $n$. The concentrations of electrons in the conduction band and holes in the valence band are designated with $n_{c}$ and $n_{v}$, respectively. One of the reason of the non-monotonic effect is thought as decrease of $p_{2}$ during the excitation due to the domination of electron capture (transition $a$ ) relative to hole capture (transition $b$ ). At high doses, stronger electron capture case is provided with the decreasing behavior of $n_{v} / n_{c}$ with dose. Authors state that it is possible to observe the nonmonotonic effect for the case of $p_{2} \ll p_{1}$ which implies that the non-radiative recombination center is dominate over the radiative center. The other necessary two conditions were reported as: (i) Recombination coefficient for free electrons with holes in non-radiative center must be smaller than retrapping coefficient of free electrons into the trapping center. (ii) Concentration of the electron trapping states must be smaller than that of nonradiative hole centers $\left(N<P_{1}\right)$. All in all, the decreasing behavior of the radiative center with dose exists due to the competition between free electron and free hole captures by this center.

The other possible reason for non-monotonic effect is thought as the competition between the radiative and nonradiative recombination centers during heating for high doses. The main point of this idea is related by authors to the case that occupancy of the non-radiative center shows a faster increase relative to that of radiative centers. This situation results with an increase of the TL intensity at low doses during heating and then a decrease at high doses after reaching to a maximum. Similar nonmonotonic dose dependence has been observed previously on some materials like $\mathrm{LiF}[10], \mathrm{Al}_{2} \mathrm{O}_{3}: \mathrm{C}[11,12]$ and $\mathrm{LiF}: \mathrm{Mg}$ [13]. 


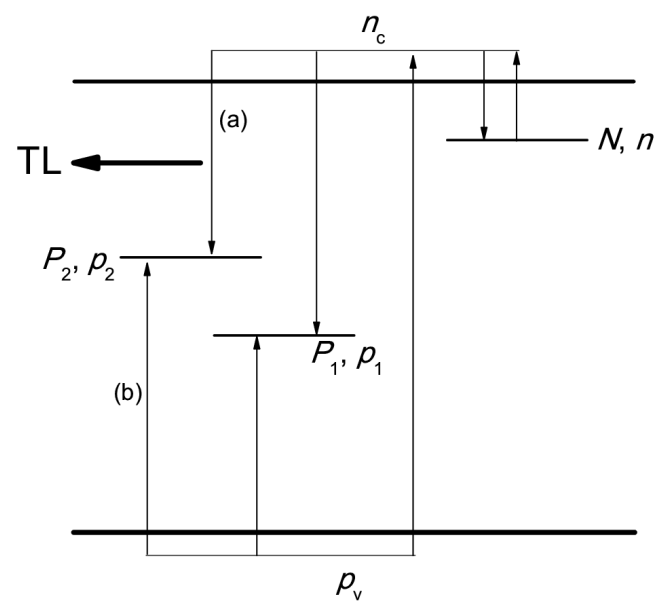

Fig. 3. Energy level diagram showing main transitions during excitation and heating.

Thermal activation energies of the trapping centers were calculated using curve fitting, initial rise and peak shape methods. TL intensity $\left(I_{\mathrm{TL}}\right)$ is related to heating rate $(\beta)$, activation energy $\left(E_{t}\right)$ and temperature $(T)$ by the expression valid for the case of first-order kinetics [14]:

$$
I_{\mathrm{TL}}=n_{0} \nu \exp \left(-\frac{E_{t}}{k T}-\int_{T_{0}}^{T} \frac{\nu}{\beta} \exp \left(-E_{t} / k T\right) \mathrm{d} T\right),
$$

in which $n_{0}$ is the initial concentration of trapped charge carriers, $\nu$ is the attempt-to-escape frequency and $T_{0}$ is the starting temperature of heating process. Curve fitting is a method based on the fitting of the experimental TL glow curve using a software. The details of the curve fitting method were reported in our previous work [15]. Fitting studies gave successful results for the case of first order of kinetics. Figure 4 shows the experimental (open circles), fitted (solid line) and deconvoluted (dash-dotted) curves corresponding to each trapping center with activation energies of $0.82,0.91$, and $0.99 \mathrm{eV}$ (Table I). Since the studied crystals were not intentionally doped, these centers are thought to originate from anion vacancies caused by nonstoichiometry and/or stacking faults, quite possible to exist in layered TlGaSeS due to the weakness of the van der Waals forces between the layers [16].

After completing the fitting process and determining the $E_{t}$ and $T_{m}$ values, we can calculate the attempt-toescape frequency $(\nu)$ by following the expression [17]

$$
\nu=\frac{\beta E_{t}}{k T_{m}^{2}} \exp \left(\frac{E_{t}}{k T_{m}}\right) .
$$

Moreover, capture cross-sections $\left(S_{t}\right)$ of the revealed traps can be determined using the relation [17]:

$$
S_{t}=\frac{\nu}{N_{v} v_{t h}}
$$

where $N_{v}$ is the effective density of states in the valence band and $v_{t h}$ is the thermal velocity of a free hole. The calculated values of $\nu$ and $S_{t}$ for each trapping centers are given in Table I.

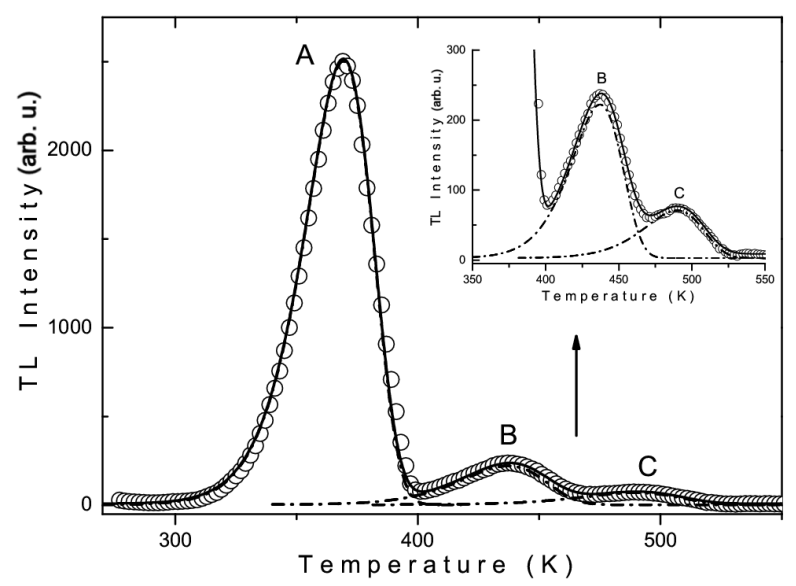

Fig. 4. Experimental TL curve of TlGaSeS crystal with heating rate of $5.0 \mathrm{~K} / \mathrm{s}$ and for $28.6 \mathrm{~Gy}$ radiation dose. Open circles are experimental data. Solid curve shows total fit to the experimental data. Dashed curves represent decomposed peaks.

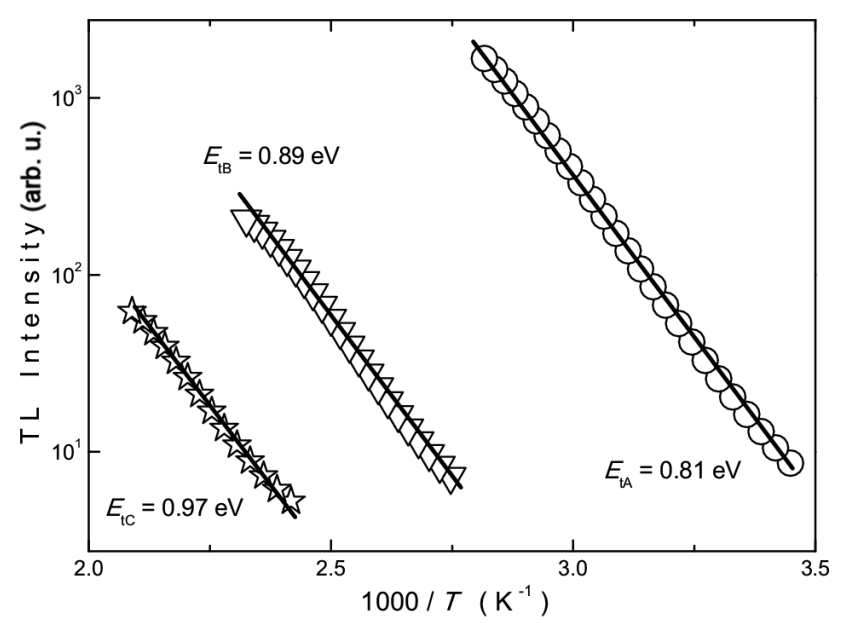

Fig. 5. TL intensity vs. 1000/T. The open shapes present the experimental data and lines represent the theoretical fit using initial rise method.

Activation energies of the centers were also calculated using initial rise method which is one of the main analysis methods used in the analysis of the TL curves [15]. The strength of this method comes due to its usage in both of order of kinetics. Integral in Eq. (1) is very small when the charge carriers began to excitation from trap level to non-localized bands. Initial rise method is applied on the region of the peak up to $\approx 10 \%$ of its maximum intensity. Since, TL intensity in this region is proportional to $\exp \left(-E_{t} / k T\right)$, the plot of $\ln \left(I_{\mathrm{TL}}\right)$ versus $1 / T$ presents a straight line with a slope of $-E_{t} / k$. Figure 5 shows the corresponding plots (open shapes) and their linear fits (solid lines). The activation energies of the centers were calculated as $0.81,0.89$, and $0.97 \mathrm{eV}$ from the slopes. The agreement between the results of initial rise and curve fitting methods is an indication of the accuracy of the activation energy value and first order of kinetics obtained from curve fitting analysis. 
TABLE I

The trapping center parameters of observed levels of TlGaSeS crystal.

\begin{tabular}{|c|c|c|c|c|c|c|c|}
\hline \multirow[b]{2}{*}{ Peak } & \multirow[b]{2}{*}{$T_{m}[\mathrm{~K}]$} & \multicolumn{3}{|c|}{$E_{t}[\mathrm{meV}]$ by method } & \multirow[b]{2}{*}{$\mu_{g}$} & \multirow[b]{2}{*}{$S_{t}\left[\mathrm{~cm}^{2}\right]$} & \multirow[b]{2}{*}{$v\left[\mathrm{~s}^{-1}\right.$} \\
\hline & & curve fitting & initial rise & peak shape & & & \\
\hline $\mathrm{A}$ & 369.2 & 0.82 & 0.81 & 0.81 & 0.42 & $1.2 \times 10^{-16}$ & $3.9 \times 10^{10}$ \\
\hline B & 437.4 & 0.91 & 0.89 & 0.89 & 0.41 & $1.0 \times 10^{-17}$ & $4.9 \times 10^{9}$ \\
\hline $\mathrm{C}$ & 490.0 & 0.99 & 0.97 & 1.00 & 0.42 & $8.0 \times 10^{-18}$ & $4.7 \times 10^{9}$ \\
\hline
\end{tabular}

Peak shape method was also applied on the TL glow curve to support the above-given results. Peak shape method is an easy analytical technique in which three parameters $\tau=T_{m}-T_{l}, \delta=T_{h}-T_{m}$ and $w=T_{h}-T_{l}$ are used to calculate the activation energy. $T_{l}$ and $T_{h}$ correspond to low and high half-intensity temperatures, respectively. $E_{t}$ value is found from the average of energies defined as $[15]$ :

$$
\begin{aligned}
& E_{\tau}=\left[1.51+3.0\left(\mu_{g}-0.42\right)\right] k T_{m}^{2} / \tau \\
& \quad-\left[1.58+4.2\left(\mu_{g}-0.42\right)\right] 2 k T_{m}, \\
& E_{\delta}=\left[0.976+7.3\left(\mu_{g}-0.42\right)\right] k T_{m}^{2} / \delta, \\
& E_{w}=\left[2.52+10.2\left(\mu_{g}-0.42\right)\right] k T_{m}^{2} / w-2 k T_{m},
\end{aligned}
$$

where $\mu_{g}$ is equal to $\delta / w$ and was predicted as 0.42 and 0.52 for first and second order of kinetics, respectively. Peak shape analysis resulted for peaks A, B and C with activation energies of $0.81,0.89$, and $1.00 \mathrm{eV}$ and $\mu_{g}$ values of $0.42,0.41$, and 0.42 , respectively (Table I). The values of $\mu_{g}$ support the result of first order of kinetics obtained from curve fitting method.

\section{Conclusion}

TlGaSeS crystals were studied by thermoluminescence experiments in the high temperature range of 280-720 K. Three TL peaks nearly at $\approx 370,437$, and $490 \mathrm{~K}$ were observed in the TL spectra. Thermal activation energies of the centers associated with these peaks were found as $0.82,0.91$, and $0.99 \mathrm{eV}$ from curve fitting analysis. Initial rise and peak shape methods were also resulted with closer values. Dose dependence of the shallowest center showed non-monotonic effect in which TL maximum intensity increased with dose up to a certain dose and then decreased at higher doses. This interesting dose dependence has been discussed under the light of a reported model which explains this dependence by considering the competition between the radiative and non-radiative recombination centers during excitation and heating stages of the TL process.

\section{References}

[1] D. Muller, H. Hahn, Z. Anorg. Allg. Chem. 438, 258 (1978).

[2] K.A. Yee, A. Albright, J. Am. Chem. Soc. 113, 6474 (1991).

[3] K.R. Allakhverdiev, Solid State Commun. 111, 253 (1999).

[4] A.M. Panich, J. Phys. Condens. Matter 20, 293202 (2008).

[5] I. Guler, N.M. Gasanly, J. Korean Phys. Soc. 51, 2031 (2007).

[6] N.M. Gasanly, J. Alloys Comp. 509, 4205 (2011).

[7] T. Yıldırım, N.M. Gasanly, Mater. Chem. Phys. 118, 32 (2009).

[8] S. Delice, N.M. Gasanly, Mod. Phys. Lett. B 28, 1450133 (2014).

[9] J.L. Lawless, R. Chen, D. Lo, V. Pagonis, J. Phys. Condens. Matter 17, 737 (2005).

[10] V.K. Jain, S.P. Kathuria, A.K. Ganguly, J. Phys. C Solid State Phys. 8, 2191 (1975).

[11] M.S. Akselrod, S.W.S. McKeever, M. Moscovitch, D. Emfitzoglou, J.S. Durham, C.G. Soares, Radiat. Prot. Dosim. 66, 105 (1996).

[12] N.A. Larsen, L. Bøtter-Jensen, S.W.S. McKeever, Radiat. Prot. Dosim. 84, 87 (1999).

[13] C.C. Crittenden, P.D. Townsend, J. Gilkes, M.C. Wintersgill, J. Phys. D Appl. Phys. 7, 2410 (1974).

[14] R. Chen, Y. Kirsh, Analysis of Thermally Stimulated Processes, Pergamon Press, Oxford 1981.

[15] M. Isik, K. Goksen, N.M. Gasanly, H. Ozkan, J. Korean Phys. Soc. 52, 367 (2008).

[16] V. Capozzi, Phys. Rev. B 28, 4620 (1983).

[17] R. Chen, S.W.S. McKeever, Theory of Thermoluminescence and Related Phenomena, World Sci., Singapore 1997. 